Kalpa Publications in Engineering
Volume 4, 2022, Pages 9-19
Proceedings of International Sym-
posium on Applied Science 2021

\title{
Studying the Influence of Illumination Design in Quality Inspection on Vaccine Vials Production Line Using Computer Vision
}

\author{
Sy Hieu Dau ${ }^{1,2, *}$, Quang My Han Doan ${ }^{1,2, *}$, Chiu Hy Ta ${ }^{1,2}$, Nguyen An \\ Khang Le ${ }^{1,2}$, Nguyen Thanh Dat Khau ${ }^{1,2}$ \\ ${ }^{1}$ Department of Biomedical Engineering Physics, Faculty of Applied Science, Ho Chi Minh City \\ University of Technology (HCMUT), 268 Ly Thuong Kiet Street, District 10, Ho Chi Minh City, \\ Vietnam. \\ ${ }^{2}$ Vietnam National University Ho Chi Minh City, Linh Trung Ward, Thu Duc District, Ho Chi \\ Minh City,Vietnam. \\ dausyhieu@hcmut.edu.vn, han.doanquang@hcmut.edu.vn
}

\begin{abstract}
In the industrial context, there are key factors that directly affect the system's efficiency. Higher demands for both quantity and quality in today's market call for constant research and development of technologies for automating production and quality control. Machine vision is a solution to increase speed and accuracy in defect detection. However, applications from machine vision are only effective if there is good data input. This is the reason why a machine vision system, needs high-quality input images from a well-designed illumination system. These illumination systems are designed to highlight faults in products. Therefore, the images obtained will provide optimized data for easier image processing thus directly increase the processing speed, accuracy, and overall system performance. To achieve this goal, this paper presents a few approaches to enhance and optimize images by implements illumination techniques into a miniature model of pharmaceutical bottle assembly line using machine vision as the inspector block. In this paper, we will evaluate the critical needs of using customize illumination system for quality inspection on an assembly line.
\end{abstract}

Keywords: Assembly line, Automatic Optical Inspection, Computer Vision, Image processing, Inspection, Illumination, LED lamp, Machine Vision, Optics, Vaccine.

\footnotetext{
${ }^{*}$ Corresponding author
} 


\section{Introduction}

In the line of production, the key target is to find an optimized solution to solve the problem between quantities and qualities. By manually checking product quality, we are limited by the abilities of human workers, affecting the number of products that can be inspected during a period of time. This leads to a large cost in labor and allows a large margin of errors.

Thanks to many developments in technology, machine vision now brings high accuracy, high stability and efficiency despite the low cost, it gives many advantages when implements in automation [1]. Computer vision has demonstrated its superiority in accuracy and stability compared to human workers over time. However, to achieve consistently accurate results, obtaining quality images must be the priority [2].

The illumination system will be desgin base on the product's physical properties and the determined defects that may occur in the production process. The light source and illumination methods will be chosen accordingly to highlight the defects for inspection. The camera and capture mode will be decided depends on the inspection requirements. These choices play an important role in the performance of the inspection block on the whole.

This paper chooses vaccine vials, a widely uses instrument in healthcare practice, as our inspecting product. Vaccine quality inspection has been in high demand, especially in number and accuracy because of the pandemic and our large population. However, the vaccine is a difficult product to inspect because the vial has a glass body with a reflective surface, inside contains transparent liquid inside and the vaccine's label is on a curve surface, this will make a normal light set up unable to produce quality images for inspection.

The purpose of this study is to offer better accuracy, speed, and a wider range of options when choosing to inspects vaccine vials without the human work. Our inspection checklists include cosmetic inspection, fill level, tip/cap inspection, label inspection, closure integrity, and the vials themself.

The illumination system is a combination of different illumination methods to inspect the vaccine in different sections. However, this illumination system is fit to our miniature model of a production line to inspect a finished product, on a real production line, the system will be modified to fit the requirements.

\section{Materials and Methods}

\section{1 Materials for System}

Camera and lens. The image acquisition system throughout this paper is composed of $5 \mathrm{M}$ resolution camera (2448 x $2048 \mathrm{px})$ and a C-Mount $16 \mathrm{~mm}$ lens to capitalize the high resolution of the camera. The physical size of each pixel is $3.45 \mu \mathrm{m} \times 3.45 \mu \mathrm{m}$, the field of view-FOV of this system is $9 \mathrm{~cm} \times 6 \mathrm{~cm}$ and the distance from the camera to the vials is $7.5 \mathrm{~cm}$. Mono image acquisition mode is used because of the ability to reduce the optical abbreviation [3].

LED and LED Controller. We use Luxeon LED $1 \mathrm{~W}$ in various lights for different application methods, each light had an operating current about 300mA-350mA. Recent studies have realized the advantages of LED PWM instead of continuous LED current [4]. The authors use a 24V PWM LED controller with a stable current release mechanism. It can change the pulse width and currents in a 
flexible manner to ensure uniform luminous intensity of the acquired image. The width can range from few dozens to a few hundred micro seconds currents [5]. The pulses width is synchronized with the camera shutter speed, therefore, reduce LED active time, heat generation is also minimized and increase the life span of LED.

\section{2 Dome Light}

Dome Illumination uses scattered light bounced from a rough surface. The light scattered in many directions will illuminate the subject's surface with the same luminous intensity at every angle. This method is intended to avoid strong reflections regardless of whether the surface to be illuminated may be convex or concave or made of strongly reflective material (metal surface, aluminum packaging surface, etc.). The images obtained by this method of illumination have a bright uniform and shadowfree field. However, the scattering surface needs to be customized to each type of product to achieve optimal results [6].

The dome light has a hollow cylindrical shape, $13 \times 11.3 \mathrm{~cm}$ with the inner radius of $5 \mathrm{~cm}$. It had the same geometrical congruence with the shape of the vaccine vial so that the light from each side will evenly cover the surface of the glass vial.

The inner surface is covered with a red filter, when white light passes from LED to the lamp, only red light is reflected, the rest of the colors either passed through or are sabsorbed so that the lamp emits only red light. The red light emitted by the lamp is absorbed by the green and black sections of the label and reflected by the white sections, giving images the best contrast possible. While the shorter wavelength will give us a higher resolution [7], we also need to check for the green color of the label. The absorption of the red color on the green label contributes to the color accuracy check on the bottle label. In addition, the location of the capture hole ensures the subject is in the center, avoid the image distortion that often appears at the edges of images.

\section{3 Dark Field (DF) and Backlight (BL) Illumination Light}

DF Illumination is the application of the light scattering effect to highlight the unusual texture on a uniform surface. Light sources are positioned so that only the rays scattered from anomalies can reach the camera. The resulting DF image will be a bright contour of the irregular detail on a dark, flat

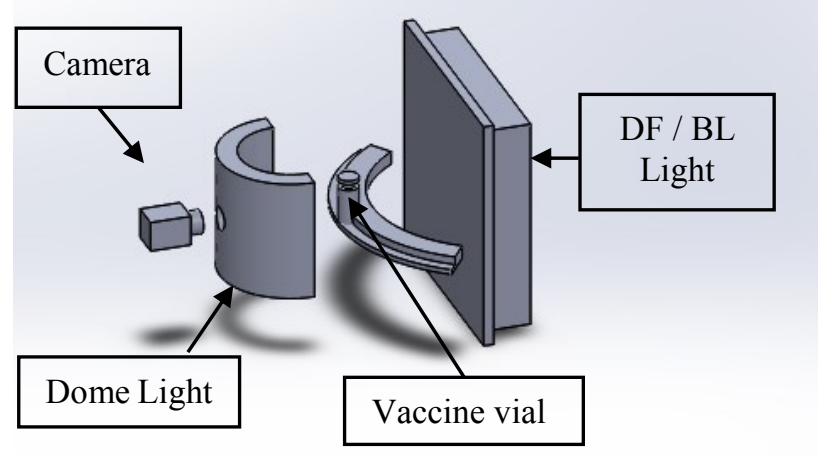

Figure 1: Schematic diagram of the illumination system. 
background. This method is very suitable for clarifying errors that are small in size or whose depth is not too great compared to the surface. The direction of the light source will be at a low angle relative to the surface and out of the area of the surface reflected angle to the camera. The wavelength is decided based on the size of the defect area and the lens in use.

BL Illumination is suitable for objects made from materials that allows light to pass through (glass, transparent liquids, etc.), using a source of light placed behind the objects. This method provides images with high contrast, clearly defined edges, suitable for measuring the size and shape of the subject.

This is a hybrid light that merged DF and BL methods. Combine with using the PWM from the LED controller, this setup allows switching between two modes consecutively in milliseconds. For that reason, the system can capture images at a faster speed with the subject's position in both modes will be almost the same, thereby minimizing the error and improving the efficiency of image processing.

\section{Results and Discussion}

\subsection{Assessment of the effectiveness of the BL Illumination}

BL method creates a good contrast in the region capable of strong light absorption and vice versa. This facilitates error detection in some regions where the light absorption and transmission zones are located adjacent to each other on the object [8].

Liquids and glass are materials that allow light to pass through, however, at the peak of the curve surface of the water and the vial, they have a tangent line from the object to the camera. Light rays that comes from the BL are bent from the normal path and do not reach the camera, results in dark edges in the images. Because of this, it is possible to identify and distinguish significant regions on the images obtained by these dark edge images in cases where manufacturers want to measure and check the fill level (Figure 2). 
Images taken in ambient light compares to images taken with BL illumination, at the same position on the image, images taken in ambient light conditions (Figure 2a), resulting in blurred images, unclear edges and noises (Figure 2b), caused by reflections and scattering from ambient light. The background of the images with ambient light is not uniformed, the image should have a low signal-to-noise ratio
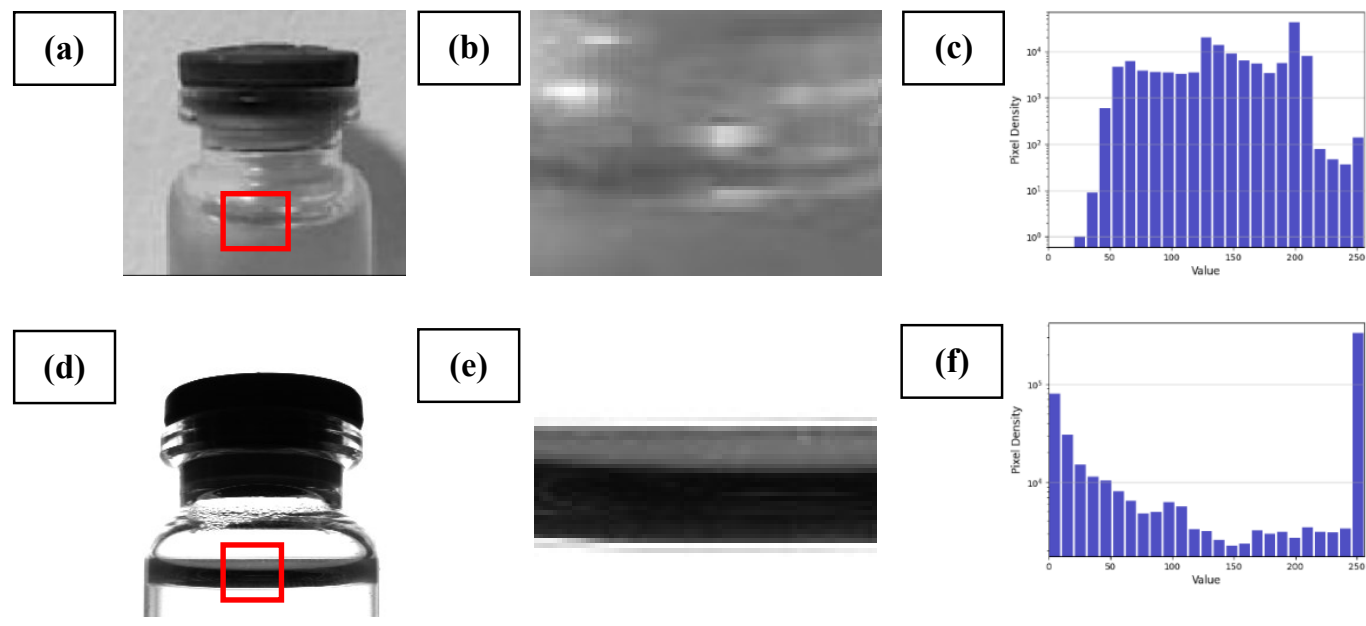

Figure 2: Images and corresponding histogram taken with ambient light (a) and images taken with BL (c).

(SNR). The contrast is low, and is almost impossible to isolate the water level accurately to analyze the results. On the other hand, images taken with the BL illumination method (Figure 2c) produce images with a clear contrast of the water level compared to the surrounding area, the brightness value of the pixels at the edge of the water level relative to the background has a high deviation. This method also creates a uniform background because of the BL, thus increate the SNR ratio. However, because of surface tension, the water level forms a concave plane, this allows an amount of light rays to reach the camera, hence why at the upper part of the water level, a small section is brighter than the rest.

The histogram of the image with ambient light shows the pixel's brightness is scattered evenly across the $\mathrm{X}$-axis. This indicates that the contrast between the water level area and the glass is low, making it difficult to perform water level error detection by image processing methods.

The histogram of the image with BL methods shows a clear section of bright and dark pixels. Therefore, the contrast of the water and the bottle is clear, water level error detection can be done simply by applies threshold in image segmentation. Moreover, the amount of data processed on the image will be reduce, the average processing time from our image processing algorithms is 0.06 seconds for water level errors.

\subsection{Assessment of the effectiveness of the DF Illumination}

DF illumination has a small irradiation angle that focuses on the edge of the object, creating an enhanced effect at the edge of the object. This facilitates detection of defects at the edges and cracks appearing on the surface of the product. 
With this method, the vial's edge forms a single thin line, with high contrast with the background (Figure 3a). Meanwhile, the plane of the BL is much larger than that of the vial, more light rays pass from the light to the body of the vial, so that its walls has a much thicker and translucent border in the image (Figure 3c).

When the subject is placed in front of the light, the received image will be closest to the real object size. Additionally, since the surface of the vial is a curved plane that allows light to pass through with a different refractive index than air, this plane refracts light traveling from the base of the lamp to the camera. Because the distance from the lamp is quite far $(7.5 \mathrm{~cm})$, images of the vial's edge is a virtual image [9]. In conclusion, to measure the vial's size, DF is the appropriate method.

This method, however, results in sharper and higher contrast edge images than the $\mathrm{BL}$ method. However, the water level is not closed at the wall of the vial because the wall structure curves inward, so the light transmission in this area is less than before (Figure 3a). This makes the bounding process of the water level impossible. Therefore, we prefer the image results of the BL method (Figure 3c).

Overall, both DF and BL methods create a good contrast of the water level compared to the surrounding image area. However, in the DF image, due to surface tension, the water surface creates a concave plane. The light at the bottom of the vial is then reflected in two directions from the highest point and the lowest point of the concave, create a double border water level. This paper will use the lower border water level to compare with the neck of the bottle, thereby assessing the water level in the vial (Figures 4-6) [10].
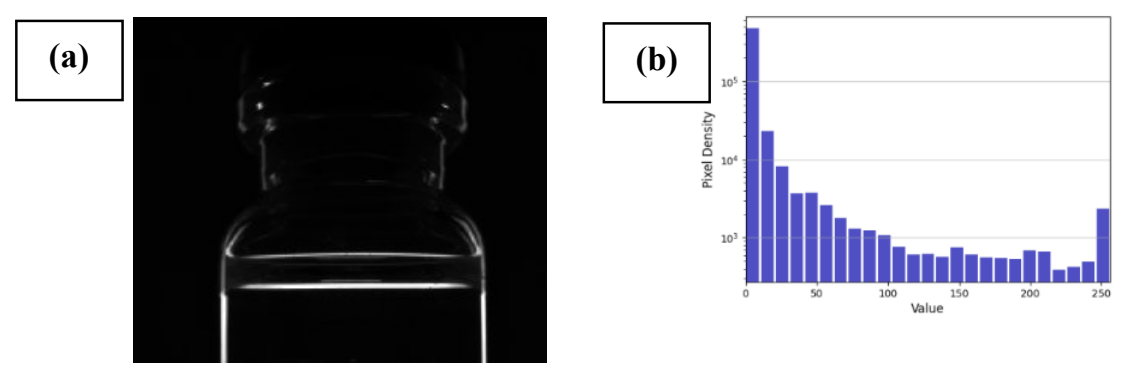

\section{(c)}
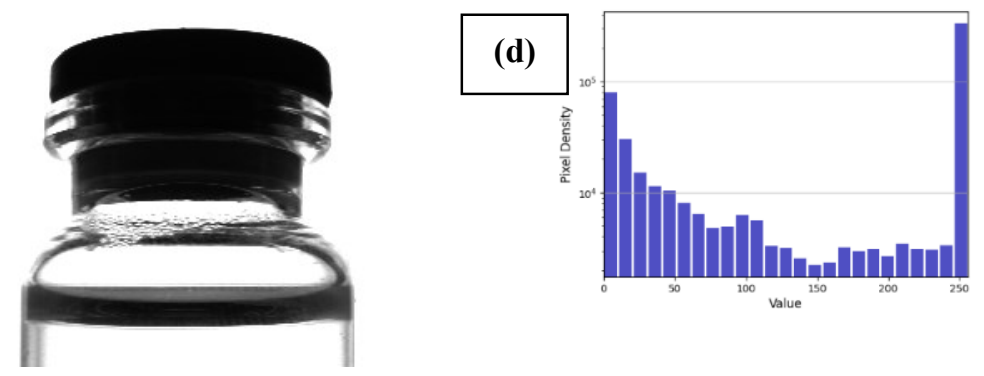

Figure 3: Images and their corresponding histogram of DF Illumination (a-b), BL Illumination (c-d). 
(a)
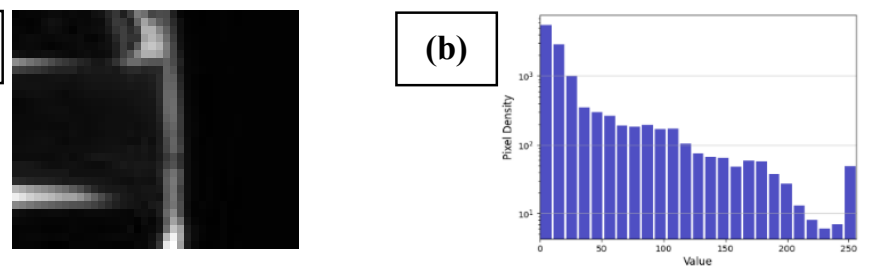

(c)

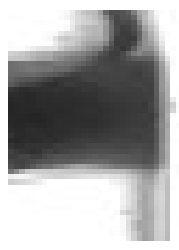

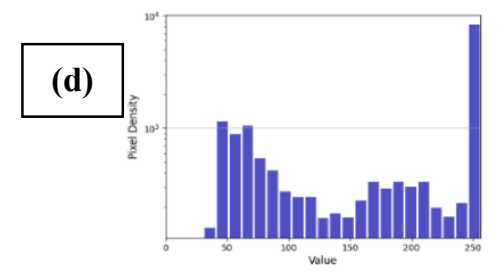

Figure 4: Enlarge detail and corresponding histogram of DF Illumination (a-b) and BL Illumination (c-d) (Figure 3).
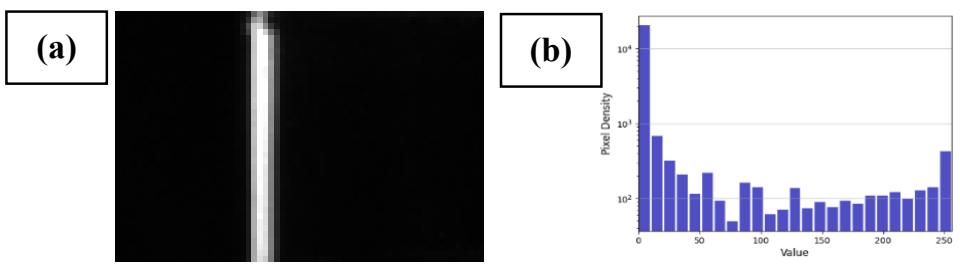

(c)

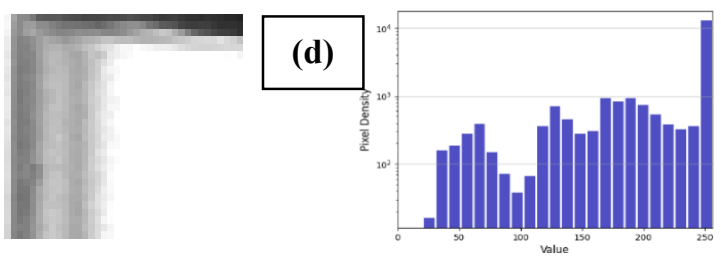

Figure 5: Enlarge detail and corresponding histogram of DF Illumination $(a-b)$ and BL Illumination (c-d) (Figure 3). 

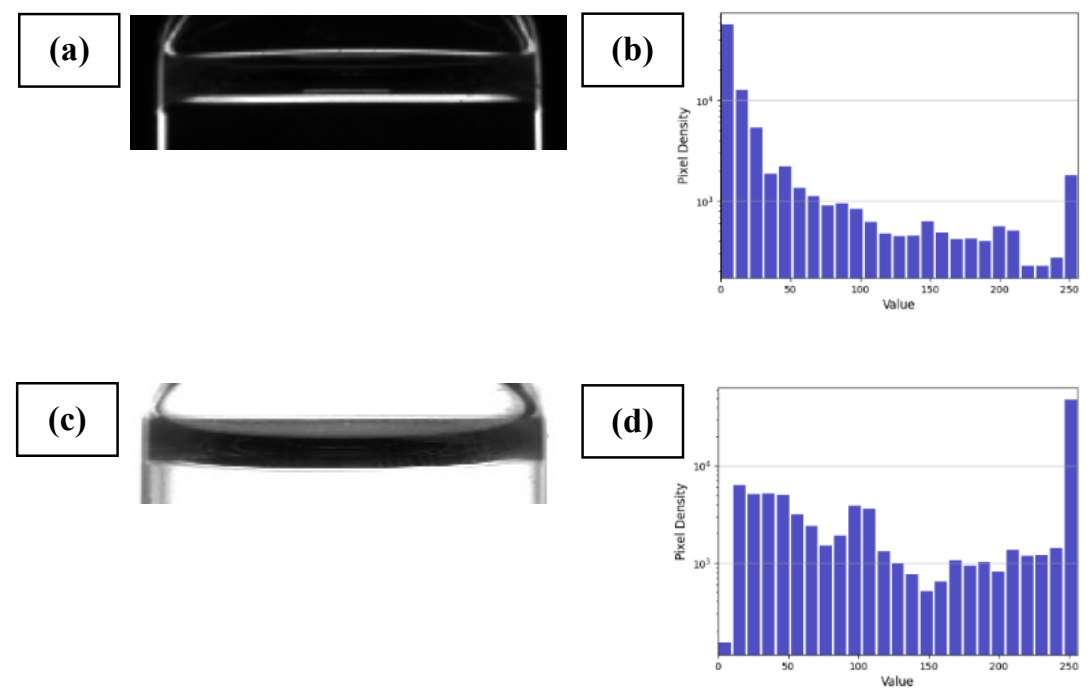

Figure 6: Enlarge detail and corresponding histogram of DF Illumination (a-b) and BL Illumination (Figure 3).

Beside the detection on water level, cap closure is another factor that affects the quality of the vaccine. In order to detect opened cap, DF methods cannot highlight the cap's edge since the cap is made from rubber, a non-reflective material (Figure 7a). However, with BL method we can see the unusual placements of the cap. Because the cap is made from a non-transparent material, light rays from the BL were blocked, therefore create the defined dark shape of the cap on a bright background (Figure $7 b)$.
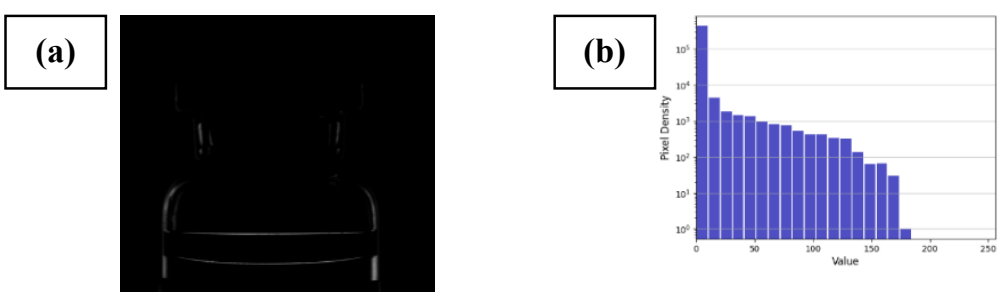

(c)
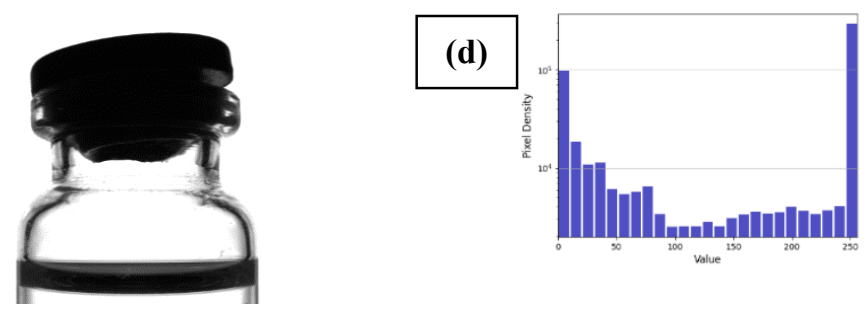

Figure 7: Leaking vial cap on vaccine with DF Illumination (a-b), BL Illumination (c-d). 
During the process of industrial production, the vial may get dirty or smudges from the handling belt. In DF images, those smudges are not highlighted because they do not reflect light as water surface, mean the detection result is not affected (Figures 8a). In this case, BL image may lead to a false detection since these smudges block the light, hence it will appear as a dark area (or boundary) in our images, which is similar to the water level (Figure 8b).
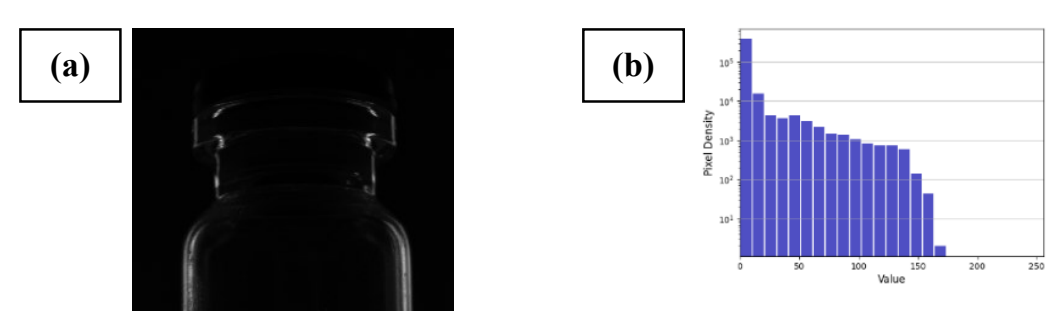

\section{(c)}
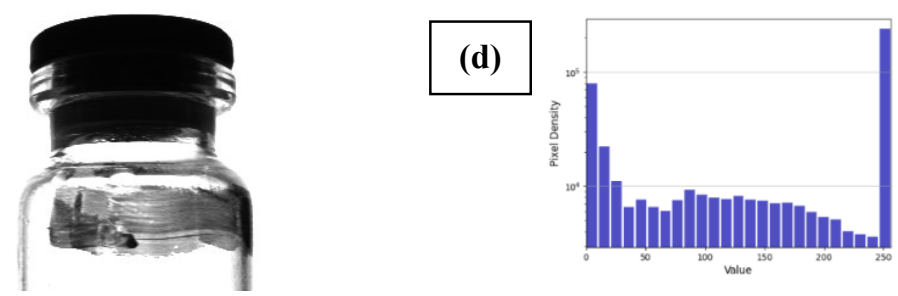

Figure 8: False water level image with DF Illumination (a-b), BL Illumination (c-d).

By combining DF and BL method, we can simultaneously check for different defects at the same position in images. Since DF illumination only enhances the boundary of the water level and BL gives a sealed image of the water level, we can eliminate the cases false conclusion as above. We have built an algorithm that compares and checks the results of image analysis between the two methods, eliminating the case of water level confusion.

\section{3 Assessment of the effectiveness of the Dome Illumination}

In the normal ambient light setting, details on the label have low contrast with blurry edges (Figures 9a-b). However, Dome Illumination creates a uniform brightfield with high contrast and clear edge (Figures 9e-f). Takes only Figure 9b, because ambient lights have no uniform brightfield, the label is not of the same brightness even in the same section results in a scattered pixel value along the $\mathrm{x}$-axis of the histogram (Figure 9c). While in Figure 9e, the red light had been absorbed by the green labels, create a dark background in contrast with the white letters and results in a clear distinction between the lettering and the background on the histogram. A simple thresholding in image processing can easily separate these two elements. Therefore, this method suitable to detects errors on the label or reading barcode if necessary. This lighting technique is also suitable for various structure of the vaccine 
vial, thanks to the principle of dome illumination, the design of the light lamp can be changed to match the structure of the object.
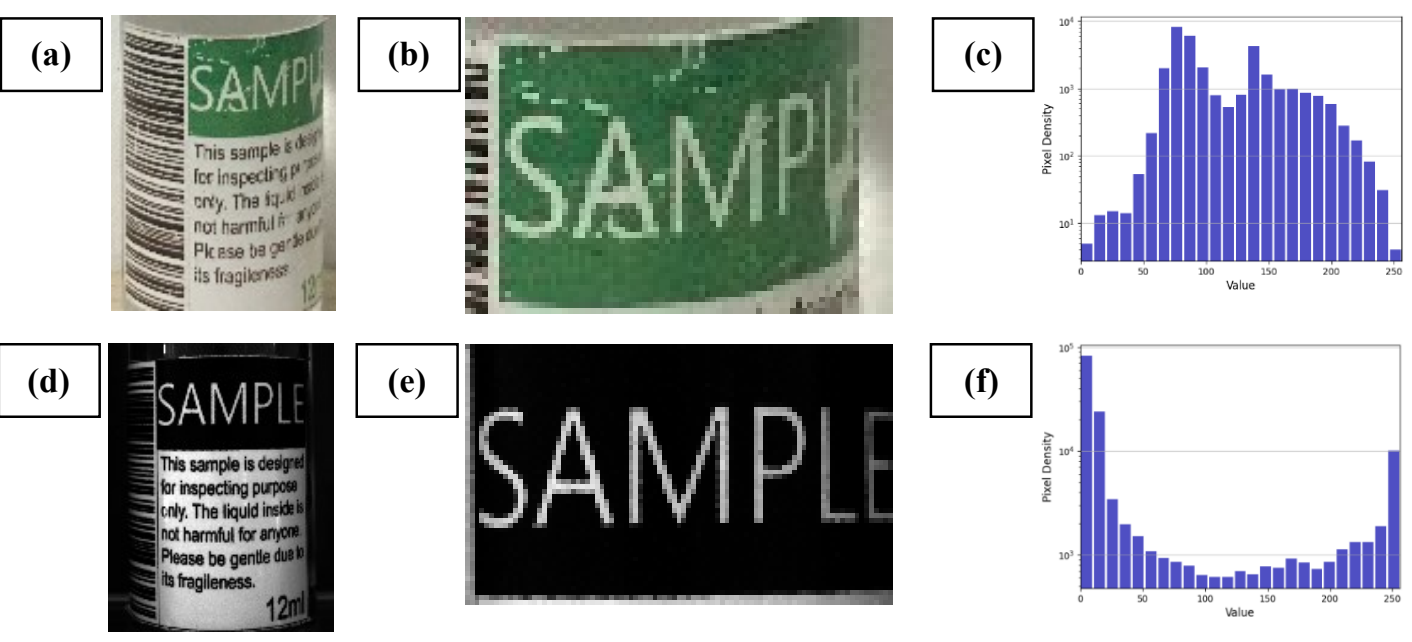

Figure 9: Images of bottle label with ambient light (a-c), Dome Illumination (e-f) and their corresponding Histogram.

\section{Conclusions}

In this study, we have designed an effective way to improve image quality and optimize data input in each image. The illumination systems have successfully eliminated disadvantages from ambient light and highlighted defects. Images obtained from the custom illumination systems have better properties for images processing. Uniformed field of light in all of images gives a consistent clear view of images. Most of the background details have also been remove and boundaries are clearly defined for easier object detection in later phases. The advantages of this illumination systems including better images quality regardless of hardware equipment (i.e high resolution camera, lens), this means most defects will show up in the images as long as the defects are not smaller than the current camera resolution.

Additionally, the application of LED and LED PWM has reduced unwanted digital noise from the external environment, improve life span of the device and avoid overheating in normal cases. This reduces time in the entire inspection block, while improving the efficiency and accuracy in the output stage. This illumination system can support an inspection block to detect easy, minimal defects and can be modified to fit to each manufactures need. Some improvements can be made with better choice of LED regarding spatial light distribution, we can use LED with broader spatial distribution for better field of light in BL method while use a narrower spatial distribution for better focus angle in DF method. 


\section{Conflicts of Interest}

The authors declare no conflicts of interest.

\section{Acknowledgement}

This research is funded by Ho Chi Minh City University of Technology (HCMUT) - VNU-HCM under grant number SVCQ-2020-KHUD-53. We would like to thank Ho Chi Minh City University of Technology (HCMUT), VNU-HCM for the support of time and facilities for this study. The authors declare that they have no conflict of interest.

\section{References}

[1] S. D. K. M. P. F. Oleksandr Semeniutaa, "Towards increased intelligence and automatic improvement in industrial vision systems," in 11th CIRP Conference on Intelligent Computation in Manufacturing Engineering (CIRP ICME'17): Innovative and Cognitive Production Technology and Systems, Ischia, Italy, 2017.

[2] A. P. F. S. W.-H. H. H. S. a. J. K. N. Rahmatov, "Machine learning-based automated image processing for quality management in industrial internet of thing," International Journal of Distributed Sensor Networks, pp. 1-11, 2019.

[3] "Chromatic and Monochromatic Optical Aberrations," Edmunds Optics.

[4] C. M. U. P. C. W. a. Z.-S. H. W.-K. Lin, "Led strobe lighting for machine vision inspection," in Int. Symposium on Next-Generation Electronics(ISNE), 2013.

[5] M. B. Jack Ganssle, "Pulse Width Modulation," in Embedded System Dictionary, 2003, pp. 103-104.

[6] I. Jahr, "Lighting in Machine Vision," in Handbook of Machine Vision, 2006, pp. 73203.

[7] J. E. Greivenkamp, "Chromatic Aberration," in Field Guide to Geometrical Optics, SPIE, 2004, p. 68.

[8] S. Z. Y. G. Q. H. Li Fu, "Medicine Glass Bottle Defect Detection Based on Machine Vision," in Chinese Control And Decision Conference (CCDC), Nanchang, China, 2019.

[9] E. Optics, "Edmund Optics on Silhouetting Illumination in Machine Vision," 2019.

[10] C. K. M. a. J. D. C. K. J. Pithadiya, "Machine vision based liquid level inspection system using ISEF edge detection technique," in 10th Proc. of the Int. Conf. and Workshop on Emerging Trends in Technology (ICWET), 2010. 